\title{
Medical Image TeXture Segmentation USINGRANGE FILTER
}

\author{
Amir Rajaei, LalithaRangarajan and ElhamDallalzadeh \\ Department of Studies in Computer Science, Mansagangothri, \\ University of Mysore, Mysore, India \\ rajaei80amir@gmail.com, lali85arun@yahoo.co.in, \\ elhamdallalzadeh@gmail.com
}

\begin{abstract}
Medical image segmentation is a frequent processing step in image understanding and computer aided diagnosis. In this paper, we propose medical image texture segmentation using texture filter. Three different image enhancement techniques are utilized to remove strong speckle noise as well enhance the weak boundaries of medical images. We propose to exploit the concept of range filtering to extract the texture content of medical image. Experiment is conducted on ImageCLEF2010 database. Results show the efficacy of our proposed medical image texture segmentation.
\end{abstract}

\section{KEYWORDS}

Medical Image Texture Segmentation, Image Enhancement, Texture Filter, Range Filter

\section{INTRODUCTION}

Computer Aided Diagnosis (CAD) is an effective tool for analyzing the particular anatomical structure of medical images leading to better diagnosis and decision-making. The number of medical images has grown significantly in the recent years. These images are very important for clinical diagnosis, localization of pathology, study of anatomical structure, treatment planning, evolution of therapy, computer integrated surgery, surgical planning, post surgical assessment and abnormality detection. It is well-established fact that segmentation plays an important role in anatomical structure recognition. Segmentation is used to extract the anatomical structure in medical images.

There exist many methods for automatic and semi-automatic medical image segmentation. However, medical image segmentation may fail to segment well the anatomical structure, the region of interest. Unknown noise, poor image contrast, inhomogeneity and weak boundaries existing in medical images have made the segmentation process extremely difficult. Further, medical images contain complicated structures.

The techniques such as thresholding[12], region growing[13], fast greedy algorithm[5], Fuzzy Cmean (FCM) and statistical models[14], active contour model [4], watershed segmentation[9,10] and clustering[8] have been proposed for medical image segmentation. Felzenszwalb and Daniel [1] proposed Minimum Spanning Tree (MST) for segmentation of medical images. MST is one of 
the graph-based segmentation methods that it is computationally efficient for capturing perceptually the important aspects of image regions. However, it is prone to cause the problem of over segmentation with some long and narrow redundant areas between two regions. In [2], authors describe an improved segmentation algorithm based on Minimum Spanning Tree (MST). To overcome the problem of over segmentation, an adaptive neighbour mode is defined by adding links between non-neighbour pixels of an image. They explored their proposed segmented method on three different modalities of medical images such as MR, CT and X-ray.

Gradient Vector Flow (GVF) snake model is used in image segmentation. In [3], GVF snake is proposed to overcome the inability of tracking concavityat boundary. However, noise existing in medical images may cause the boundaries of medical images not to converge.Hence, in these complicated images GVF still fails to capture object contours. To improve GVF snake model, Chuag and $\mathrm{Li}$ [11] proposed a new downstream algorithm based on Extended Gradient Vector Flow (E-GVF) for segmentation of multi-objects.In [7], they focused on texture features as well as region growing algorithm to automate segmentation of the MR images. Co-occurrence texture features and semi-variogram texture features are extracted from the image and then the seeded region growing algorithm is run on these feature spaces. With a given region of interest, a seed point is automatically picked up based on three homogeneity criteria. Medical ultrasound images are poor in contrast and they have strong speckle noise. Hence, traditional image segmentation methods fail to segment the ultrasound images satisfactorily. In [6], the ultrasound images are segmented using texture feature and graph cut method. The texture feature parameters are obtained according to the Gary level Co-occurrence Matrix (GLCM).

From the literature survey it is observed that a number of segmentation methods are applied on only specific anatomical structure such as the use of GVF for segmentation of kidney images. Moreover, the proposed methods are exploited on particular medical imaging modalities. Furthermore, the well-known characteristics of medical images such as unknown noise, poor image contrast, inhomogenity and weak boundaries affect the anatomical structure resulting in poor segmentation. In this direction, we propose an algorithm to segment medical images that include various anatomical structures belonging to different medical imaging modalities such as X-ray, CT, MR, US, PET, PET-CT, Xr-Angio, NM, Micro. Besides, the poor characteristics of medical images are moderated to have accurate medical image segmentation. The characteristic of regions in an image are analyzed by their texture content.

The rest of the paper is organized as follows. Image filtering is discussed in section 2. Our proposed algorithm for texture segmentation of medical images is explained in section 3. Section 4 gives the details of the experiments followed by the results. The paper is concluded in section 5 .

\section{IMAGE FILTERING}

Filtering is perhaps the most fundamental operation of image processing. The term filtering can be defined as the value of the filtered image at a given location. It is a function of the values of the input image in a small neighbourhood of the same location. Filter operations can be used to sharpen or blur images, to selectively suppress image noise, to detect and enhance edges, or to alter the contrast of the image. The filters use the local statistical variations in an image to reveal elements of the image texture.

\subsection{Analyzing the Texture of an Image}

Texture analysis refers to the characterization of regions in an image by their texture content. Texture analysis attempts to quantify intuitive qualities described by terms such as rough, 
smooth, silky or bumpy as a function of the spatial variation in pixel intensities. In this sense, the roughness or bumpiness refers to variations in the intensity values or gray levels.

Texture analysis is used in a variety of applications, including remote sensing, automated inspection and medical image processing. Texture analysis can be used to find the texture boundaries and texture segmentation. Texture analysis can be helpful when objects in an image are more characterized by their texture than by intensity and hence, traditional thresholding techniques cannot be used effectively. The texture analysis functions such as range filtering, standard deviation filtering and entropy filtering, filter an image using standard statistical measures. These statistics can characterize the texture of an image. They provide information about the local variability of the pixels intensity values in an image. In the areas with smooth texture, the range values in the neighbourhood around a pixel will be small and similarity,the range values are large in the areas of rough texture.

The texture functions all operate in a similar way. They define a neighbourhood around the pixel of interest calculate the statistic for that neighbourhood and use the computed statistic value as the value of the pixel of interest in the output image. The example shown in Figure 1 illustrates how the range filtering function operates on a simple matrix. In this example, the value of element B $(2,4)$ is calculated from A $(2,4)$. Range filtering function use $\mathrm{m}$ by $\mathrm{n}$ pixels, in this example $3 \times 3$, neighbourhood around the pixels.

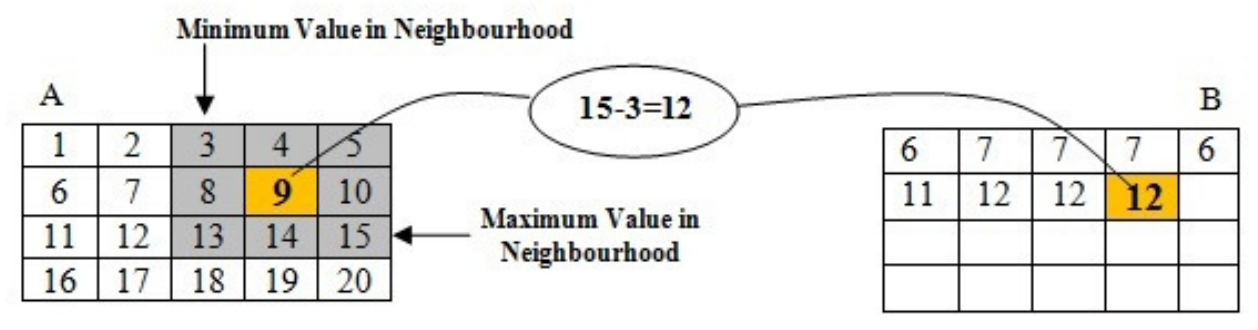

Figure1.Range filtering function

\section{Proposed Method}

Image texture includes the local spatial pattern, scale and magnitude of brightness variations, smoothness or roughness of the image. The output image can be used as the basis for further image analysis such as image texture segmentation. Hence, in this paper we exploit 3 different kinds of image enhancement technique to remove the strong speckle noise existing in a medical image as well image edge enhancement. The details are explained in section 3.1. We propose texture filtering to segment medical image texture. We propose to utilize the range filtering for segmentation.Image texture segmentation using range filtering is discussed in section 3.2.

\subsection{Pre-processing}

The contrast of medical images is very low and these images have strong speckle noise. We apply $2 \mathrm{D}$ adaptive noise-removal, median and $2 \mathrm{D}$ order-statistic filtering to remove the noise and enhance the weak boundaries of medical images. 2D adaptive noise removal is a low-pass filter on gray scale images. 2D adaptive noise removal uses a pixel wise adaptive wiener method based on the statistics estimated, mean and standard deviation, from a local neighbourhood of size $\mathrm{m}$ by $\mathrm{n}$ for each pixel. Result of image enhancement using $2 \mathrm{D}$ adaptive noise removal is shown in Figure 2(b). To remove 'salt and pepper' noise existing in medical images, we use median filtering. Median filtering is a nonlinear operation which is very effective to simultaneously 
reduce noise and preserve edges. Each output pixel contains the median value in $\mathrm{p}$ by $\mathrm{q}$ neighbourhood around the corresponding pixel of the input image. Figure 2(c) shows removal of "salt and pepper" noise from a medical image using median filtering. The order statistic filter is then applied to enhance the regions of an anatomical structure. The 2D order-statistic filtering replaces each pixel of an image by the $n^{\text {th }}$ order element in the sorted set of neighbours of size $r$ by $\mathrm{s}$ specified by the nonzero elements in domain. Image edge enhancement using $2 \mathrm{D}$ order-statistics filtering is shown in Figure 2(d). In this paper, the medical image pre-processing step is done by the three discussed image enhancement functions as shown in Figure 2(e).

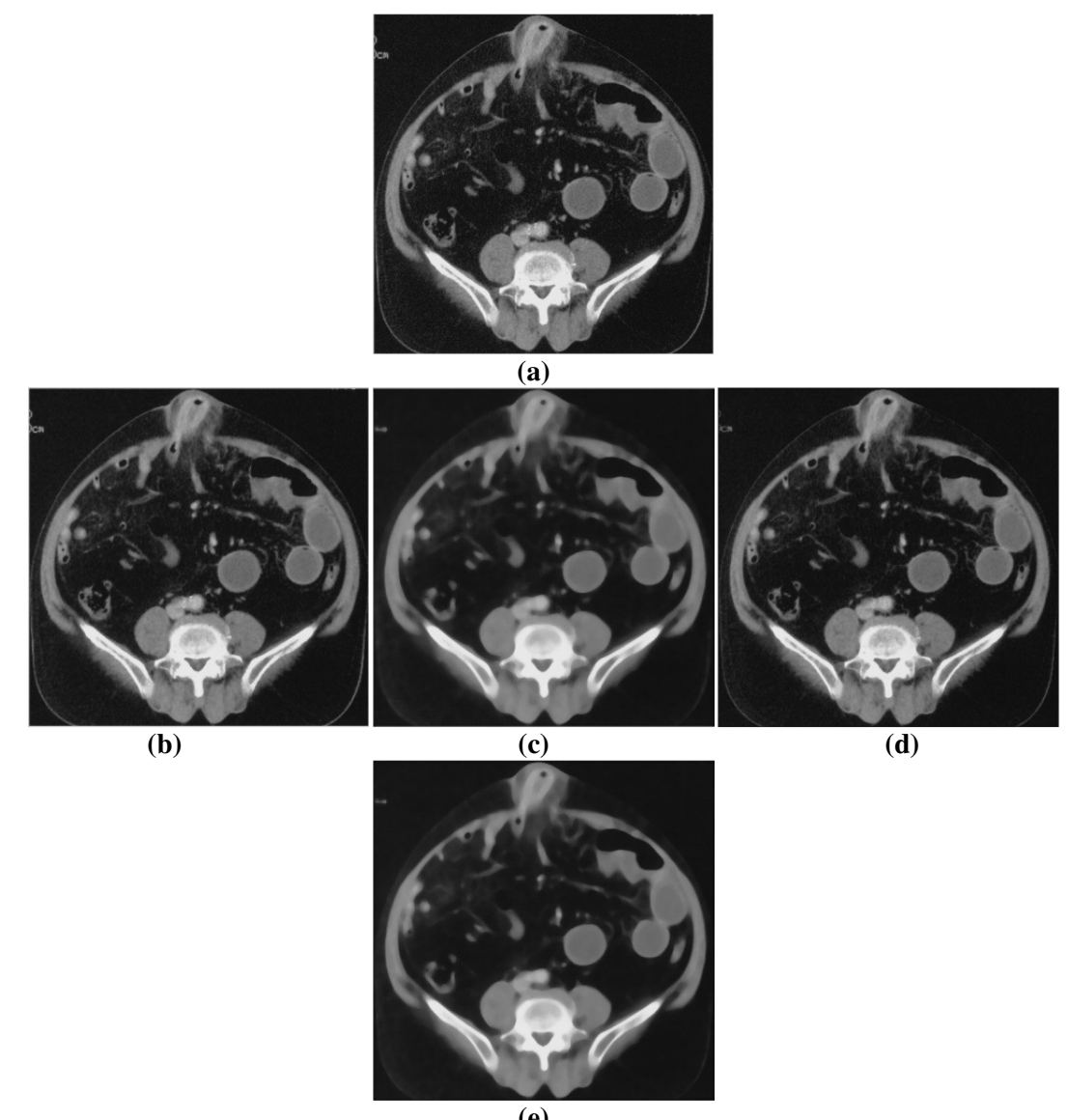

Figure2.(a) Original image. (b) Applied adaptive noise removal on an image.

(c) Applied median filtering on an image. (d) Applied order-statistic filtering on an image.

(e) Pre-processedimage.

\subsection{Image Texture Segmentation}

Medical image segmentation is a critical task for analyzing the structural content of the images. Surgical planning, early disease detection and 3D visualization can be provided for the physicians by proper image segmentation. Medical image texture segmentation can be widely applicable to evaluate an area of body that is not externally visible. The texture filter function can detect the texture regions of a medical image. It can be argued that there is a little variation in the gray level values of the background of medical imagessince the background is smooth. Hence in the foreground the surface contours of the anatomical structure exhibit more texture. Therefore, the foreground pixels of medical images have more variability and thus higher range values. Range 
filtering is one of the texture analysis methods that filter an image. Range filtering makes the edges and contours of the anatomical structures of medical images become more apparent.Consequently, the range filtering is explored for medical image texture segmentation as explained in the following sub section.

\subsubsection{Range Filtering}

A local sub range filtering uses the statistical sub range of the pixel intensities within the window. The range distance is often used in statistics as a measure of the sample variation. Edges are typically characterized by discontinuities in mean intensity. If the variations existing among the local intensity values are low then the local range distance is small. Similarly, the local range distance is large if a region has large discontinuities in intensity values. Hence, range filtering is able to detect pixel intensity values of the edges within a window.The output of the range filtering is the difference between maximum and minimum range values of the filtered window. The range values in the filtered window are multiplied by a constant value to provide strong edges. Local range filtering tend to have short calculation time as it operates on only a small number of input for each output pixel.Moreover, range filtering can have better segmentation through creating a structure element to extract the neighbourhood for the local range of values. Hence a structuring element is created to extract the neighbourhood for the local range of values. Figure 3 illustrates the range filtered medical image using the defined structure element.

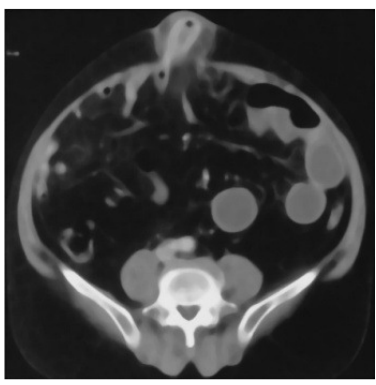

(a)

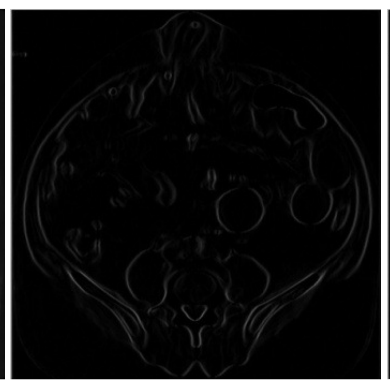

(b)

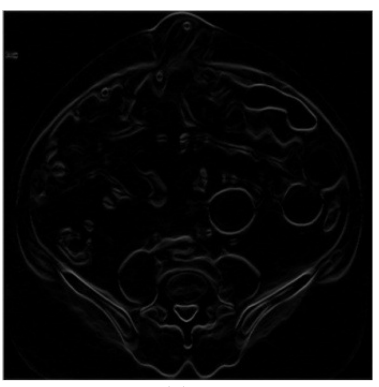

(c)

Figure 3.(a) Pre-processedimage. (b) Range filtered medical image using horizontal linestructure. (c) Range filtered image using vertical line structure.

\section{EXPERIMENTAL RESULTS}

\subsection{Image Dataset}

In this paper, ImageCLEF2010 database is used for segmentation of medical images. Experiments are conducted on different medical imaging modalities having different sizes. The medical imaging modalities are nine in number namely, Compute Tomography (CT), Magnetic Resonance (MR), Ultrasound (US), Nuclear Medicine (NM), Xr-Angio, Positron Emission Tomography (PET), PET-CT, Micro and X-ray. Medical images include various anatomical structures and image orientation.

\subsection{EXPERIMENTAL SETUP}

Medical images contain strong speckle noise. To remove noise and smooth the images we have applied 2D adaptive noise removal using neighbourhood of size 3 by 3 . Moreover, medical images suffer from "salt and paper" noise. We remove the existing 'salt and paper' noise using median filtering. Each output pixel contains the median value of 9 by 9 neighbourhoods 
surrounding the pixel of the input image. Further, It is well known that the content of medical images are inhomogeneous having weak boundaries. We use $2 \mathrm{D}$ order-statistic filtering by the $3^{\text {rd }}$ order element in the sorted set of neighbours of size 3 by 3 in domain.

We have exploited range filtering using 'line' structure element of size $1 \times 2$ and $2 \times 1$ in horizontal and vertical directions respectively. Next, we have applied sum operation on the images extracted using range filtering in both horizontal and vertical directions. Figure 4(a) shows the added image obtained from the medical images illustrated in Figure 3(b) and 3(c) respectively. In addition, to improve the extracted edges of the ranged filtered medical image shown in Figure 4(a) we scale the image by multiplying with the fix value of ' $k$ '. In this experiment, the value of ' $k$ ' is set to 20 empirically. Figure 4(b) illustrates the scaled range filtered medical image.

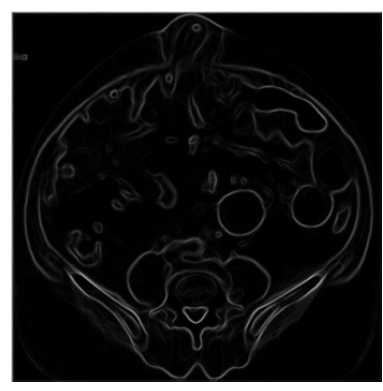

(a)

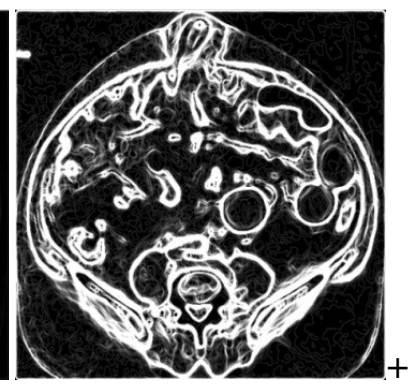

(b)

Figure 4.(a) Range filteredmedical images after filtering in horizontal and vertical directions are added. (b) Scaled range filtered medical image.

Next, we have used 'clean' morphological operation to remove all detected isolated pixels. Figure 5 demonstrates the segmentation of the anatomical structure medical image using our proposed texture segmentation method.

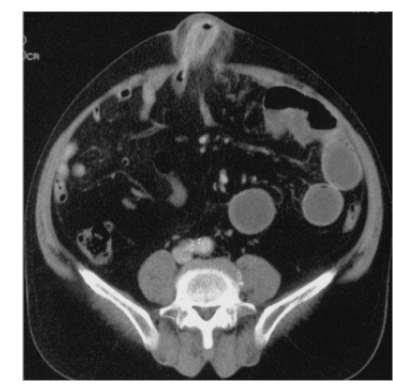

(a)

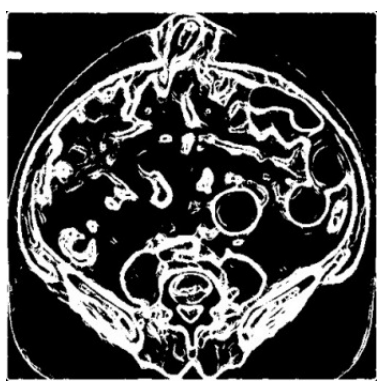

(b)

Figure 5.(a) Original image. (b) Medical imagesegmentation.

In this paper, experiments are conducted on nine different medical imaging modalities having different anatomical structures. The proposed medical image texture segmentation using range filtering is shown in Figure 6.

\section{ConClusion}

In this paper, 2D adaptive noise removal, median and 2D order-statistic filtering are applied to remove strong speckle noise and enhance the weak boundaries of medical images. We segment various anatomical structures of different medical imaging modalities using texture filtering. The 
range filtering is exploited for medical image texture segmentation.Results show the efficiency, simplicity and robustness of medical image texture segmentation
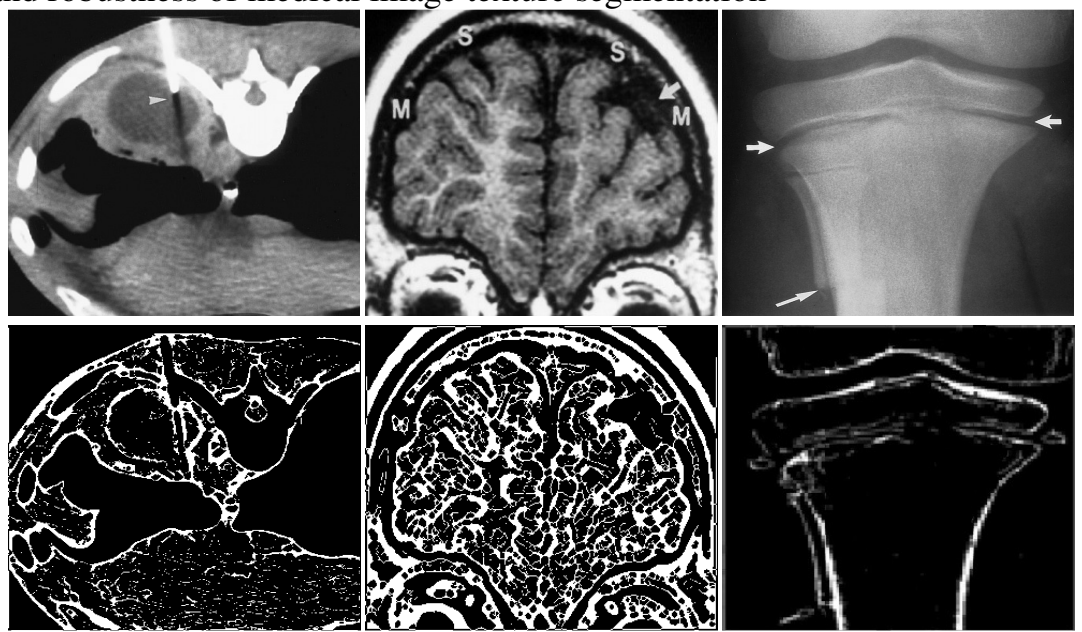

(a)CT

(b)MR

(c)Xray
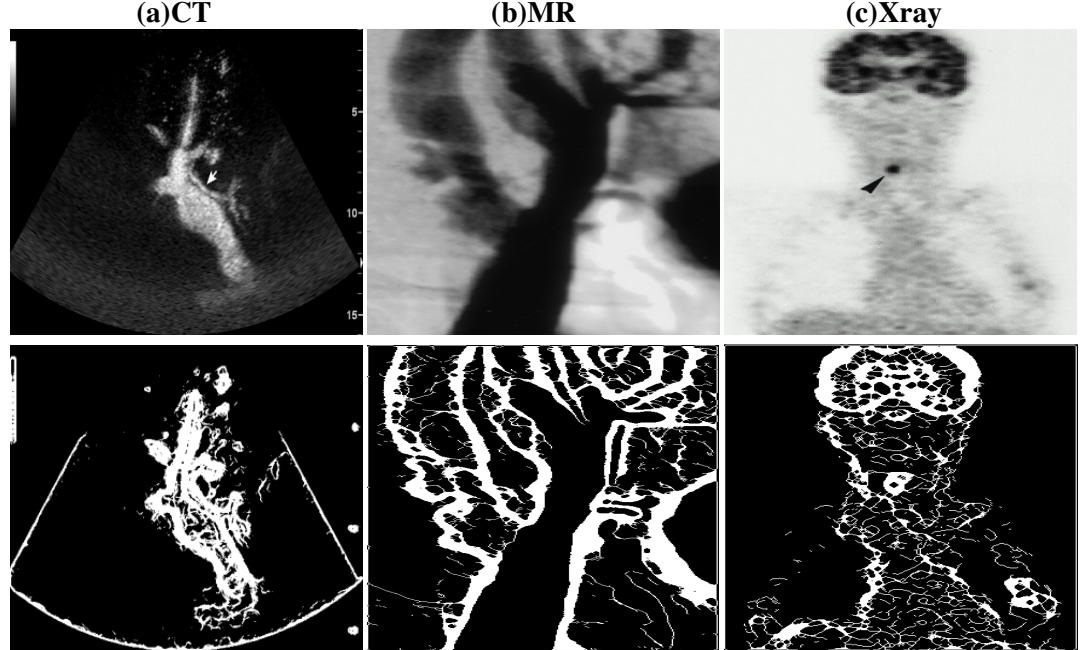

(d)US

(e)Xr-Angio

(f)PET
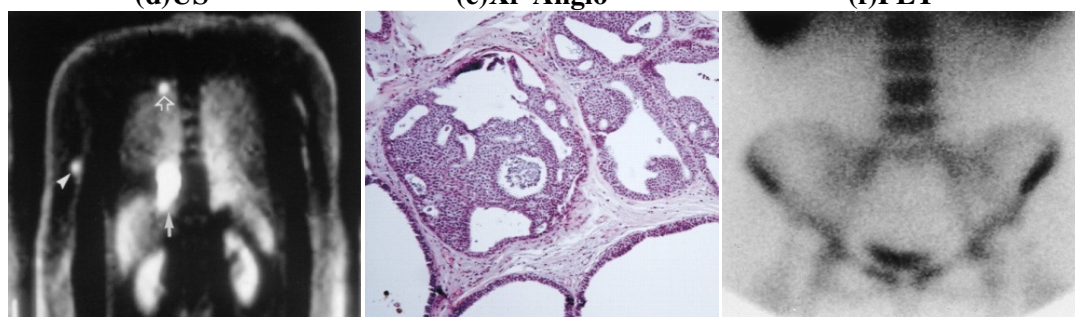

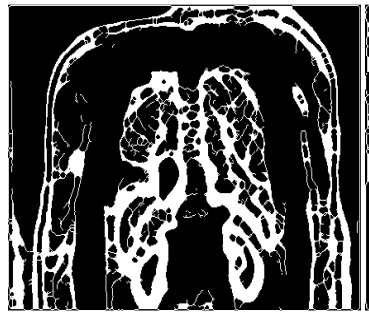

(g)PET-CT

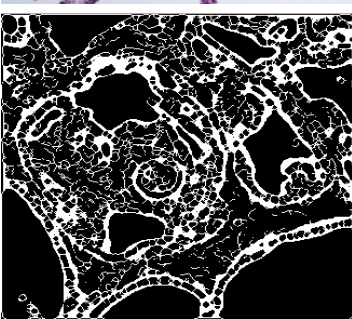

(h)Micro

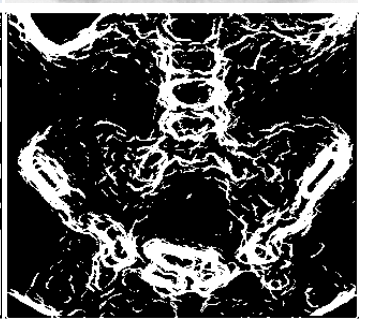

(i)NM

Figure 6.(a-i)Segmented anatomical structures of differentmedical imagingmodalities. 


\section{REFERENCE}

[1] Pedro F. Felzenszwalb and P, Daniel, (2004) "Efficient Graph-Based Image Segmentation," International Journal of Computer Vision, Vol.59 (2), pp. 167-181.

[2] Y. Lu, Y. Quan, Z. Zhang and G. Wang, (2009) "MST Segmentation for Content-Based Medical Image Retrieval", International Conference on Computational Intelligent and Software Engineering, pp.1-4.

[3] J. Cheng, R. Xue, W. Lu and R. Jia,(2008) "Segmentation of Medical Images with Canny Operator and GVF Snake Model”, 7th World Congress on Intelligent Control and Automation, pp.1777-1780, chaina

[4] L.D. Cohen, I.Cohen, (1993) "Finite-element Methods for Active Contour models and Balloons for 2D and 3D Images," IEEE Transaction on Image Processing on Pattern Analysis \& Machine Intelligence Vol.25, pp.1131-1147.

[5] D.J.Williams, M.Shah, (1991) "A Fast Algorithm for Active Contours and Curvature Estimation," CVGIP: Image Understanding, vol.55, pp.14-26, Jan.

[6] Z. Chang-ming, G. Guo-chang, L. Hai-bo, S. Jing and Y. Hualong, "Segmentation of Ultrasound Image Based on Texture Feature and Graph Cut", International Conference on Computer Science and Software Engineering, pp.795-798.

[7] J. Wu, S. Poehlman, M. Nosewrthy and M.V. Kamath, (2008) "Texture Feature based Automated Seeded Region Growing in Abdominal MRI Segmentation”, International Conference on Biomedical Engineering and Informatics, pp.263-267.

[8] TianD, Fan L,( 2007) “A Brain MR Images Segmentation Method Based on SOM Neural Network”, in the First International Conference on Bioinformatics and Biomedical Engineering, pp.868-689.

[9] Adalsteinsson D, Sethian JA, (1995) “A Fast Level Set Method for Propagating Interfaces," Journal of Computing Physics, 118, pp.269-277.

[10] Li N, Liu M and Li Y,( 2007) " Image Segmentation Algorithm using Watershed Transform and Level Set Method," IEEE Transaction International Conference on Acoustics, Speech and Signal Processing, pp.613-616.

[11] C.Chuang, W.Lie, (2004) "A Downstream Algorithm Based on Extended Gradient Vector Fellow Field for Object Segmentation,” IEEE Transaction on Image Processing, Vol.13, pp.1379-1392, October.

[12] Suzuki H, Toriwaki J,(1991) “Automatic Segmentation of Head MRI Images by Knowledge Guided Thresholding”, Computer Medical Imaging Graph.Vol.15(4), pp.233-40.

[13] Robb RA, (2000) "Biomedical Imaging, Visualization and Analysis", edited by Wiley-Liss, USA.

[14] Hall Lo, Bensaid AM, Clarke LP, Velthuizen RP, Silbeger MS., Bezdek J, “ A Comparison of Neural Network and Fuzzy Clustering Techniques in Segmenting Magnetic Resonance Images of the Brain ”, IEEE Transaction Neural Networks, Vol.26(4), pp. 479-486. 